\title{
Validation of the neutron monitor yield functions using data from AMS-02 and PAMELA experiments, 2006-2017
}

\author{
Sergey A. Koldobskiy* \\ Oulu University, Oulu, Finland and National Research Nuclear University MEPhI, Moscow, \\ Russia \\ E-mail: sakoldobskiy@mephi.ru
}

\section{Veronica Bindi}

University of Hawaii at Manoa, Honolulu, USA

\section{Claudio Corti}

University of Hawaii at Manoa, Honolulu, USA

\section{Gennady A. Kovaltsov}

Ioffe Physical-Technical Institute, St. Petersburg, Russia

\section{Ilya G. Usoskin}

Oulu University, Oulu, Finland

\begin{abstract}
Spectra of protons and helium directly measured in space by the AMS-02 experiment for the period 2011-2017 and protons measured by the PAMELA experiment for 2006-2014 provide a unique opportunity to calibrate ground-based neutron monitors (NMs). Here we present calibration results of several stable sea-level NMs (Inuvik, Apatity, Oulu, Newark, Moscow, Hermanus, Athens) using these measured spectra. Four modern NM yield functions were verified: Mi13 (Mishev et al., JGR:SP, 2013), Ma16 (Mangeard et al., JGR:SP, 2016), CM12 (Caballero-Lopez \& Moraal, JGR:SP, 2012) and CD00 (Clem \& Dorman, SSR, 2000). The Mi13 yield function was found to realistically represent the NM response to galactic cosmic rays. CM12 yield function leads to a small skew in the solar cycle dependence of so-called NM scaling factors. In contrast, Ma16 and CD00 yield functions tend to overestimate the NM sensitivity to low-energy cosmic rays. This effect may be important for an analysis of ground level enhancements (GLEs), leading to a potential underestimate of fluxes of solar energetic particles as based on data. The Mi13 yield function is recommended for quantitative analyses of NM data, especially for ground-level enhancements. The results of this work strengthen and validate the method of the cosmic-ray variability analysis based on the NM data and yield-function formalism, and improves its accuracy.
\end{abstract}

36th International Cosmic Ray Conference -ICRC2019-

July 24th - August 1st, 2019

Madison, WI, U.S.A.

${ }^{*}$ Speaker. 


\section{Introduction}

Over the last two decades we significantly improved our understanding of the cosmic-ray (CR) physics, thanks to new generation experiments such as PAMELA [1] and AMS-02 [2].

Both experiments, among other highly appreciated results, provide important new information about low-energy cosmic rays, where the effects of solar modulation are significant. Published AMS-02 and PAMELA data covers 11 years of cosmic-ray observations: from 2006 to 2014 for PAMELA experiment and from 2011 to 2017 for AMS-02 experiment.

Galactic cosmic ray (GCR) flux is modulated by a heliospheric magnetic field (HMF), produced by the Sun, and HMF changes drastically during cycles of solar activity (11 and 22-year, 27-day or during transient events, such as coronal mass ejections or solar flares).

Traditional way to study the solar modulation of cosmic rays for the last decades since 1950's is an analysis of the neutron monitor data. Neutron monitors (NM) are integral detectors suitable for registration of secondary neutrons generated in nucleonic cascades induced by cosmic-ray particles penetrating the atmosphere. Since neutron detectors register secondary particles it is impossible to reconstruct the initial spectrum of different cosmic-ray species. But it is possible to solve the inverse problem: by assuming the spectrum of primary particles to reconstruct the NM response. The most modern way to do it is the use of the neutron-monitor yield function, using this method the NM count rate can be computed as:

$$
N\left(P_{\mathrm{c}}, h, t\right)=\sum_{j} \int_{P_{\mathrm{c}}}^{\infty} J_{j}(R, t) \cdot Y_{j}(R, h) \cdot d R
$$

where $Y_{j}(R, h)$ is the yield function of the NM (located at height $h$ ) for primary cosmic-ray particles of type $j$ (protons, helium, heavier species), and $J_{j}$ is the differential rigidity intensity of primary particles of type $j$ at the Earth's orbit but outside the magnetosphere and atmosphere. Yield function (YF) merge the processes of the particle propagation through the Earth's atmosphere and the detection response of a NM to secondary particles generated in the atmosphere.

The new AMS-02 and PAMELA data for proton spectra and the AMS-02 data for helium spectra with Bartels/Carringron rotation time resolution gives an excellent opportunity to calibrate the neutron monitors and validate yield functions, because in this case we know the initial CR spectra and the neutron monitor response experimentally. So in this work we have tested four modern yield functions and made a comparison of reconstructed solar modulation potential for both NM data and PAMELA/AMS-02 data.

In this report only a short description of obtained results is presented, for a more detailed description of methods and results please refer to $[3,4]$.

\section{Selection of data}

For this analysis we used two publication of the PAMELA collaboration with Carrington rotation time resolution proton spectrum data for 2006 - 2010 [5] and 2010 - 2014 [6] and a recent AMS-02 collaboration publication considering the proton and alpha-particle fluxes for period 2011 - 2017 with Bartels rotation (BR) time intervals [7].

NM data was taken using dedicated web-servers for Oulu (cosmicrays.oulu.fi) and Apatity (pgia.ru/CosmicRay/) NMs and using the NMDB database for other NMs. Data 
from seven NMs were considered: Inuvik (geomagnetic cutoff rigidity $\left.\mathrm{P}_{c}=0.3 \mathrm{GV}\right)$, Apatity $(0.65$ GV), Oulu (0.8 GV), Newark (2.4 GV), Moscow (2.43 GV), Hermanus (4.58 GV), Athens (8.53 $\mathrm{GV})$.

\section{Yield function calibration}

Four modern yield function were considered in this analysis: Mi13 [8], Ma16 [9], CM12 [10], CD00 [11].

For all these functions averaged count rates were calculated from the real data and reconstructed with the AMS-02 proton and helium data for the same periods using Equation 1.1.

Previously the nucleonic ratio of the heavier species (including all species with $\mathrm{z}>1$ ) to protons in the local interstellar spectrum (LIS) was taken fixed as about 0.3 of proton spectra [12]. In this work we independently from protons considered the heavier-than-proton particles for a first time by using direct measurements of various cosmic ray species spectra made by AMS-02 and other experiments. As input parameter of our model we used the measured helium spectra for each $\mathrm{BR}$, assuming that all helium is ${ }^{4} \mathrm{He}$. Heavier-than-helium species for each BR period have not been published yet, so that only time-integrated over several years spectra have been used for this study. Since the heliospheric modulation of all heavier species is expected to be similar to that of helium, because of the similar charge-to-mass ratio, $Z_{i} / A_{i}$, they can be roughly scaled to each other and their ratio to helium in rigidity is expected to be constant irrespectively of the modulation level. Therefore, for each heavier specie we assumed that the time-dependent spectrum can be obtained by scaling the measured time depended spectrum of helium.

We performed a full recalibration of the NM response and verified the validity of the yield functions using, as $J_{i}(R, t)$, the spectra of protons and helium (the latter scaled to account for heavier species, as described above) measured by AMS-02 with BR scale over 2011 - 2017. Using equation 1.1 we reconstructed the expected count rates of several stable NMs covering the range from mid to high geomagnetic latitudes using the AMS-02 spectra of protons and helium and compared these expected count rates with the measured ones.

Through this method we reconstructed the relevant scaling factor $\kappa$, defined as the ratio of the modeled to measured count rates. In Table 1 scale factors $\kappa$ are summarized for seven NMs and four YFs. The scaling factors $\kappa$ are usually slightly greater than unity, indicating that the efficiency of a NM is slightly lower than that of an ideal NM. This is related to the local environment (e.g., constructions above the NM) or electronic setup (high voltage or dead time). These close-to-unity $\kappa$ values were obtained for Mi13 and Ma16 YFs. CM12 YF systematically but not drastically differs from the unity. CD00 yield function leads to too high scaling factors, suggesting that it may overestimate the NM response.

The linear correlations between scaling factor $\kappa$ and count rate of the NMs were constructed considering all BRs and corresponding linear regression slopes $s$ were calculated.

In Figure 1 we show the regression slopes $s$ as function of the geomagnetic cutoff rigidities $P_{c}$ for four considered yield functions. One can see that the Mi13 produces no significant relation even for the high-latitude NMs. On the other hand, all other yield functions lead to a statistically significant relation between the two quantities for polar NMs, which decreases toward higher cutoff rigidities and disappears for Athens NM. The strongest relation (as high as 3.5-4\%/Hz) is for Ma16 
Table 1: Parameters (name, effective vertical geomagnetic cutoff rigidity $P_{\mathrm{c}}$ and altitude) of the used NMs along with the found scaling factors $\kappa$ (see text) for the four yield functions studied here: Mi13, Ma16, CM12 and CD00. Error bars represent the standard error of the mean $\kappa$ over all BRs.

\begin{tabular}{ccc|cccc}
\hline NM & $P_{\mathrm{c}}(\mathrm{GV})$ & Alt $(\mathrm{m})$ & $\kappa \mathrm{Mi} 13$ & $\kappa \mathrm{Ma} 16$ & $\kappa \mathrm{CM} 12$ & $\kappa \mathrm{CD} 00$ \\
\hline Inuvik & 0.3 & 21 & $1.333 \pm 0.003$ & $1.237 \pm 0.011$ & $1.533 \pm 0.005$ & $1.833 \pm 0.011$ \\
Apatity & 0.65 & 177 & $1.47 \pm 0.005$ & $1.364 \pm 0.012$ & $1.69 \pm 0.006$ & $2.021 \pm 0.012$ \\
Oulu & 0.8 & 15 & $1.199 \pm 0.003$ & $1.113 \pm 0.010$ & $1.378 \pm 0.004$ & $1.649 \pm 0.011$ \\
Newark & 2.4 & 50 & $1.311 \pm 0.006$ & $1.207 \pm 0.011$ & $1.505 \pm 0.007$ & $1.789 \pm 0.011$ \\
Moscow & 2.43 & 200 & $1.366 \pm 0.004$ & $1.256 \pm 0.009$ & $1.569 \pm 0.005$ & $1.862 \pm 0.010$ \\
Hermanus & 4.58 & 26 & $1.308 \pm 0.003$ & $1.142 \pm 0.005$ & $1.471 \pm 0.004$ & $1.677 \pm 0.005$ \\
Athens & 8.53 & 260 & $1.14 \pm 0.007$ & $0.927 \pm 0.007$ & $1.247 \pm 0.007$ & $1.348 \pm 0.007$ \\
\hline
\end{tabular}

and CD00 yield functions and quite moderate $(\approx 1 \% / \mathrm{Hz})$ for $\mathrm{CM} 12$ one. This implies that these yield functions may introduce an additional bias of up to $10 \%$ within a solar cycle, where the NM count rate varies by $1-2 \mathrm{~Hz}$ per counter, and that most of the yield functions tend to overestimate the response of a NM to low-energy cosmic rays (below several gigavolts). This leads to a positive relation between $\kappa$ and the count rate. On the other hand, the fact that the relation is absent even for polar NMs for the Mi13 yield function suggests that the low-energy part of this yield function is correct. Although this effect is not large for GCRs, within 10\%, it may be important for a study of solar energetic particle events, leading to a potential underestimate of their flux. Since all the yield functions lead to no relation for Athens NM, we may assume that all of them correctly reproduce the NM response in the rigidity range above $8 \mathrm{GV}$.

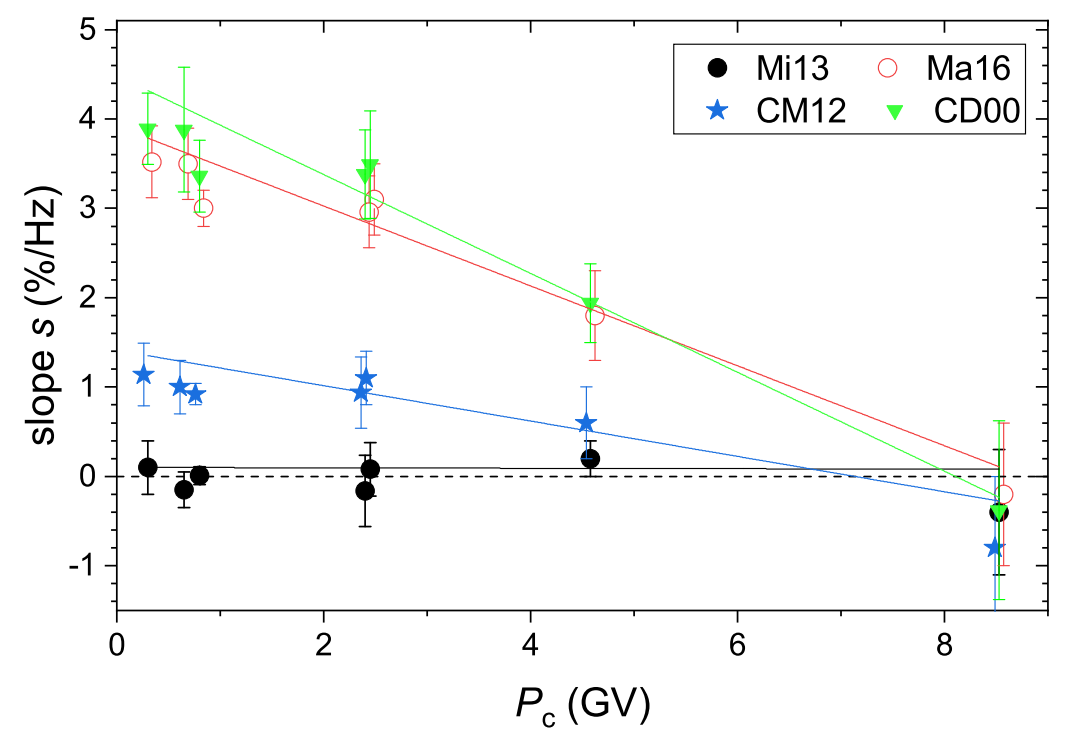

Figure 1: The slope of the linear regression between the scaling factor $\kappa$ and the count rate, along with the standard error, for different yield functions (colors as denoted in the legend) and NMs, characterized by their geomagnetic cutoff rigidity $P_{\mathrm{c}}$. 


\section{Reconstruction of the solar modulation potential}

The new AMS-02 data cover a wide range of solar modulation, from low activity in 2011 to the maximum in 2014 and again to the declining phase toward a new minimum, while PAMELA data covers the period from 2006 to 2014, covering decreasing, minimum and increasing phases of solar activity.

Transport and modulation of GCR in the heliosphere is described by the Parker transport equation [13], which includes four main mechanisms: diffusion, convection and adiabatic cooling in the radially expanded solar wind as well as drifts in HMF. In 1967 Gleeson and Axford [14] proposed an analytical solution for a simplified version of the Parker equation, which uses only one parameter describing the solar modulation strength: the solar modulation potential $\phi$. Although it has little physical sense because of the simplified assumptions, the force-field model provides useful for practical purposes parametrization of the near-Earth GCR spectrum.

Here we describe the work performed to check the validity of the force-field approximation. Spectra of protons measured for each BR were individually fitted with the force-field model to define the corresponding modulation potential $\phi$. The force-field model links the energy spectrum of GCR particles of a given type $J$ near Earth with their reference intensity outside the heliosphere, called the local interstellar spectrum (LIS) $J_{\text {LIS }}$, so that

$$
J_{i}(T, \phi)=J_{\mathrm{LIS}_{i}}\left(T+\Phi_{i}\right) \frac{T\left(T+2 T_{\mathrm{r}}\right)}{\left(T+\Phi_{i}\right)\left(T+\Phi_{i}+2 T_{\mathrm{r}}\right)}
$$

where $T$ is the kinetic energy per nucleon, $T_{\mathrm{r}}=0.938 \mathrm{GeV}$ is the proton's rest mass, and $\Phi_{i}=\phi \cdot\left(e Z_{i} / A_{i}\right)$. The exact value of the modulation parameter depends on the reference LIS, here we used a recent estimate of the proton LIS by Vos \& Potgieter [15].

The measured by AMS- 02 and PAMELA proton spectra were fitted with the force-field model (Equations 4.1) using the $\chi^{2}$ method (as described by [3]). We fitted the proton spectra in the energy range $1-30 \mathrm{GeV} /$ nucleon, since this energy interval is affected by solar modulation. The fit agrees with the data within $\pm 10 \%$ for the solar maximum period and within a few $\%$ during the ascending and descending phases of the solar cycle. During quiet periods, the quality of the fit is better than for periods with high activity. This is understandable, since solar modulation is more complicated, in particular by propagating barriers, during active than during quiet periods. Nevertheless, the fits are good even during active periods. We note that the force-field approximation is not thought to precisely reproduce the exact spectrum of particles near Earth, but only provides a reasonable parameterization for it. The obtained $10 \%$ accuracy is fairly good for practical purposes of assessing the cosmic-ray-related atmospheric effects and fully validates the use of the force-field model for applications, while it should not be applied for a detailed study of cosmic rays.

Figure 2 shows the time dependence of obtained $\phi$-value from the AMS-02 and the PAMELA data and reconstructed values from the NM network [12], which were normalized to PAMELA data for 2006-2010, and thus the new AMS-02 and PAMELA 2010 - 2014 data serves as a direct test for the reconstruction. The AMS-based values lie close to the PAMELA-based ones during the time of their overlap, that shows that the GCR proton spectra measured by both experiments are consistent. The NM-based modulation potential looks systematically too low, by 50-70 MV during the solar maximum, and agrees with the AMS-based ones for the times of moderate solar 
activity and then exceeds the fitted values by $\approx 40 \mathrm{MV}$ after 2016 . Here we also plotted the NM-based values of $\phi$ reconstructed using the NM calibration with the AMS-02 data (denoted as magneta solid line). Comparison between NM-based $\phi$-values normalized to PAMELA and AMS02 data shows the systematical error of the developed method of $\phi$ reconstruction. Namely, there is up to $20 \mathrm{MV}$ difference in reconstructed $\phi$ values using different datasets (PAMELA 2006-2009 and AMS-02 2011-2017).

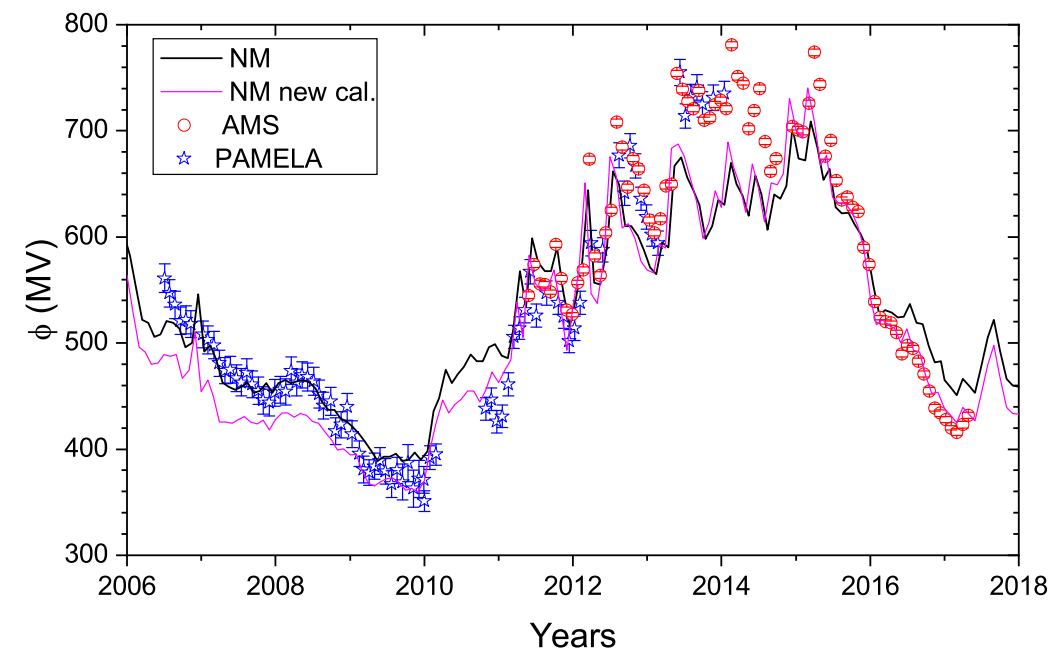

Figure 2: Values of the modulation potential $\phi$ reconstructed here from space-borne (symbols) and NMbased (solid curves) data. $\phi$-values are from [4] for AMS-02 data, [3] for PAMELA and [12] for NM-based data (black line). The $\phi$ reconstructed from the NM data with calibration to the AMS-02 dataset is shown with solid magneta curve.

\section{Conclusion}

The newly published spectra of protons and helium measured directly by AMS-02 collaboration for the period 2011-2017 for individual 27-day BR periods allowed us to perform the full calibration and verification of the ground-based NM detectors. We have presented the result of calibration of seven stable sea-level NMs (Inuvik, Apatity, Oulu, Newark, Moscow, Hermanus, Athens) using four modern NM yield functions (Mi13, Ma16, CM12 and CD00), by comparing the calculated NM count rates, on the basis of the cosmic-ray spectra measured by AMS-02 with those actually recorded for the corresponding time intervals. The Mi13 yield function was found to realistically represent the NM response to GCRs. In contrast, Ma16 and CD00 ones tend to overestimate the NM sensitivity to low-energy cosmic rays, leading to a possible bias, of the order of $5-10 \%$, for the 11-year cycle in GCR. This effect may be crucial if these yield functions are applied to an analysis of ground level enhancements, caused by solar energetic particles with much softer spectrum than GCR. In particular it may lead to a significant underestimate of fluxes of solar energetic particles as based on NM data. Accordingly, these yield functions need a revision in the lower-rigidity part. CM12 yield function also depicts a possible small overestimate of the low-energy sensitivity of a NM, leading to a few percent bias. We recommend to use Mi13 yield function for quantitative analyses of NM data. 
We have also checked the validity of the force-field approximation using AMS-02 and PAMELA data for period 2006 - 2014 and found that it indeed provides a good parametrization for the directly measured proton spectra, within a $10 \%$ uncertainty. The accuracy of the approximation is very good (within a few $\%$ ) for periods of low to moderate activity and slowly degrades to $\approx 10 \%$ for active periods.

\section{Acknowledgments}

Data of AMS-02 were obtained from NASA SPDF database http: / / www . spase-group . org/registry/render?f=yes\&id=spase://VSPO/NumericalData/ISS/AMS-02/ P27D. Data of NMs count rates were obtained from http:// cosmicrays.oulu.fi (Oulu NM), http://pgia.ru/CosmicRay/ (Apatity), Neutron Monitor Database (NMDB) and IZMIRAN Cosmic Ray database (http://cro. izmiran.ru/common/links.htm). NMDB database (www. nmdb.eu), founded under the European Union's FP7 programme (contract no. 213007), is not responsible for the data quality. Balloon and AMS-01 data were obtained from SSDC database (https://tools.ssdc.asi.it/CosmicRays/). PIs and teams of all the ballon- and space-borne experiments as well as ground-based neutron monitors whose data were used here, are gratefully acknowledged. This work was partially supported by the ReSoLVE Centre of Excellence (Academy of Finland, project no. 307411), by National Science Foundation Career Award under grant (NSF AGS-1455202); Wyle Laboratories, Inc. under grant (NAS 9-02078); NASA under grant (17-SDMSS17-0012), and by the RFBR and NRF according to the research project 19-52-60003 and MEPhI Academic Excellence Project (contract 02.a03.21.0005)

\section{References}

[1] O. Adriani et al. "Ten Years of PAMELA in Space". In: Rivista del Nuovo Cimento 40.10 (2018), pp. 473-522. arXiv: 1801.10310.

[2] M Aguilar et al. "Precision Measurement of the Proton Flux in Primary Cosmic Rays from Rigidity $1 \mathrm{GV}$ to $1.8 \mathrm{TV}$ with the Alpha Magnetic Spectrometer on the International Space Station". In: Physical Review Letters 114.17 (2015), p. 171103. arXiv: 1612.08562.

[3] Sergey A. Koldobskiy, Gennady A. Kovaltsov, and Ilya G. Usoskin. "A Solar Cycle of Cosmic Ray Fluxes for 2006-2014: Comparison between PAMELA and Neutron Monitors”. In: Journal of Geophysical Research: Space Physics 123.6 (2018), pp. 4479-4487.

[4] Sergey A. Koldobskiy et al. "Validation of the Neutron Monitor Yield Function Using Data From AMS-02 Experiment, 2011-2017”. In: Journal of Geophysical Research: Space Physics 124.4 (2019), pp. 2367-2379.

[5] O Adriani et al. "Time dependence of the proton flux measured by PAMELA during the July 2006 - December 2009 solar minimum”. In: The Astrophysical Journal 765.8pp (2013), p. 11. arXiv: 1301.4108.

[6] M Martucci et al. "Proton Fluxes Measured by the PAMELA Experiment from the Minimum to the Maximum Solar Activity for Solar Cycle 24". In: The Astrophysical Journal 854.1 (2018), p. L2. arXiv: 1801.07112. 
[7] M Aguilar et al. "Observation of New Properties of Secondary Cosmic Rays Lithium, Beryllium, and Boron by the Alpha Magnetic Spectrometer on the International Space Station". In: Physical Review Letters 120.2 (2018), p. 021101.

[8] A L Mishev, I G Usoskin, and G A Kovaltsov. "Neutron monitor yield function: New improved computations". In: Journal of Geophysical Research: Space Physics 118.6 (2013), pp. 2783-2788.

[9] P.-S. Mangeard et al. "Monte Carlo simulation of the neutron monitor yield function". In: Journal of Geophysical Research: Space Physics 121.8 (2016), pp. 7435-7448.

[10] R. A. Caballero-Lopez and H. Moraal. "Cosmic-ray yield and response functions in the atmosphere”. In: Journal of Geophysical Research: Space Physics 117 (2012), A12103.

[11] John M. Clem and Lev I. Dorman. "Neutron monitor response functions". In: Space Science Reviews 93.1-2 (2000), pp. 335-359.

[12] Ilya G Usoskin et al. "Heliospheric modulation of cosmic rays during the neutron monitor era: Calibration using PAMELA data for 2006-2010". In: Journal of Geophysical Research: Space Physics 122.4 (2017), pp. 3875-3887.

[13] E.N. Parker. "The Passage of Energetic Charged Particles through Interplanetary Space". In: Planetary and Space Science 13 (1965), pp. 9-49.

[14] L. J. Gleeson and W. I. Axford. "Cosmic Rays in the Interplanetary Medium". In: The Astrophysical Journal 149 (1967), p. L115.

[15] Etienne E. Vos and Marius S. Potgieter. "NEW MODELING OF GALACTIC PROTON MODULATION DURING THE MINIMUM OF SOLAR CYCLE 23/24”. In: The Astrophysical Journal 815.2 (2015), p. 119. 